\title{
Context-independent and context-dependent information in concepts
}

\author{
LAWRENCE W. BARSALOU \\ Stanford University, Stanford, California 94305
}

\begin{abstract}
It is proposed that concepts contain two types of properties. Context-independent properties are activated by the word for a concept on all occasions. The activation of these properties is unaffected by contextual relevance. Context-dependent properties are not activated by the respective word independent of context. Rather, these properties are activated only by relevant contexts in which the word appears. Context-independent properties form the core meanings of words, whereas context-dependent properties are a source of semantic encoding variability. This proposal lies between two opposing theories of meaning, one that argues all properties of a concept are active on all occasions and another that argues the active properties are completely determined by context. The existence of context-independent and context-dependent properties is demonstrated in two experimental settings: the property-verification task and judgments of similarity. The relevance of these property types to cross-classification, problem solving, metaphor and sentence comprehension, and the semantic-episodic distinction is discussed.
\end{abstract}

Some properties in a concept seem to come to mind on all occasions. The word "skunk" usually makes people think of the property "unpleasant smell," and "rattlesnake" usually makes people think of "poisonous." In contrast, other properties in a concept rarely seem to come to mind, and when they do, it is only in relevant contexts. For example, "basketball" rarely makes people think of "floats." However, the sentence frame "Chris used $X$ as a life preserver when the boat sank" would probably bring "floats" to mind for "basketball" when " $\mathrm{X}$ " is "basketball." In this paper, I propose there are two important types of properties associated with concepts: context-independent (CI) properties and context-dependent (CD) properties. CI properties are activated by the word for a concept on all occasions (e.g., "unpleasant smell" for "skunk"). CD properties are rarely if ever activated by the word for a concept and are only activated by relevant contexts in which the word appears (e.g., "floats" for "basketball").

CI properties form the core meanings of words. This is because they are activated by the respective word on all occasions, independent of contextual relevance. Barsalou and Bower (Note 1) have proposed that properties become automatically activated by a word after being frequently associated with it during processing.

This research was supported by Grant MH 13950 from the National Institute of Mental Health to Gordon $\mathrm{H}$. Bower and by a National Science Foundation graduate fellowship to the author. I am grateful to Gordon Bower for supporting this research and to Kathleen Hemenway, Brian Ross, Ronald Finke, Michael McCloskey, and an anonymous reviewer for excellent comments on earlier drafts. Requests for reprints should be sent to Lawrence W. Barsalou, Department of Psychology, Emory University, Atlanta, Georgia 30322.
Frequent pairings of a word and a property cause an automatized relation between them to be established in memory (also see Shiffrin \& Schneider, 1977). Barsalou and Bower (Note 1) showed that two types of properties are likely to be frequently active during the processing of a word. First, properties having high diagnosticity may often be active, since they are useful for distinguishing instances of a concept from instances of other concepts. "Gills" becomes CI for "fish" because all fish have gills and no other things do. The second type of property likely to be frequently active during the processing of a word includes properties relevant to how people typically interact with instances of the respective concept. "Edible" becomes CI for "apples" because it is central to how people typically interact with them. As shown by "edible" in relation to "apple," properties frequently relevant to human interaction can become $\mathrm{CI}$ even if they have low diagnosticity (i.e., "edible" is true of many other things).

CD properties are a source of semantic encoding variability. $C D$ properties may be represented in concepts, but they are not usually activated by encoding the respective words. Rather, these properties are activated only by relevant encoding contexts in which a word appears. Semantic encoding variability is the result of different encoding contexts of a word activating different subsets of $C D$ properties in the respective concept. This phenomenon has frequently been observed empirically (e.g., R. C. Anderson \& Ortony, 1975; R C. Anderson, Pichert, Goetz, Schallert, Stevens, \& Trollip, 1976; Barclay, Bransford, Franks, McCarrell, \& Nitsch, 1974; Tulving \& Thompson, 1973) and has been incorporated theoretically by Bower (1972) and Estes (1955, 1959). Barsalou and Bower (Note 1) suggest that CD properties are typically inactive because they have 
rarely, if ever, been processed simultaneously with their respective words. Hence, the associations between these properties and their respective words are weak or nonexistent. When such associations do not exist, various inference processes may be required to compute them. People may not have stored the fact that "fits in a suitcase" is a property of "flashlight," but they can certainly infer it. If a CD property comes to be frequently processed with a word, the property may change status and become CI. Although "wears horseshoes" may be CD for "horse" for people who are rarely around horses, it could become CI for someone during the course of learning to be a horseshoer.

Some properties are probably neither $\mathrm{CI}$ nor $\mathrm{CD}$. These may sometimes be activated by a word, although not on all occasions, and may sometimes be activated by context. Such properties may occasionally be activated by a word because of random fluctuations in the amount of activation the property receives. On other occasions, however, these properties may be activated by relevant contexts. The senses of ambiguous words can similarly not be classified as strictly CI or CD. This is because they often come to mind without context, but they are also influenced by sentence contexts in which they occur (Swinney, 1979; Tanenhaus, Leiman, \& Seidenberg, 1979). Nevertheless, the senses of ambiguous words can be viewed as concepts that contain $\mathrm{CI}$ and $\mathrm{CD}$ properties. Once an ambiguous word is disambiguated in context, the distinction between $\mathrm{CI}$ and $\mathrm{CD}$ properties becomes applicable to the concept converged upon. Consider "bear" in the sentende "The bear caught pneumonia." "Bear" and the sentence frame both converge on the mammalian sense of "bear." However, some of the properties activated for this sense are CI (e.g., "is furry," "can be dangerous") and some are CD (e.g., "can be sick," "has lungs"). Beyond acknowledging their existence, I will not further consider properties and word senses that are neither CI nor CD. Rather, the purposes of this paper are (1) to demonstrate the existence of $\mathrm{CI}$ and $\mathrm{CD}$ properties in concepts and (2) to consider the roles these property types play in various cognitive phenomena.

This paper addresses a particular aspect regarding the structure of concepts, namely, the accessibility of properties. Two theories of meaning take more extreme views on this aspect. Traditional views of semantics (e.g., Katz \& Postal, 1964) assume that the meaning of a word contains a fixed set of semantic features applicable on all occasions on which the word is used. It appears that semantic memory models usually make a similar assumption (e.g., Glass \& Holyoak, 1975; McCloskey \& Glucksberg, 1979; Smith, Shoben, \& Rips, 1974). In the terms of this paper, this approach argues that all of a concept's properties are CI. An opposing and more radical view of semantics (e.g., Olson, 1970) argues that the meaning of a word completely depends on the context in which the word is used. Specifically, the meaning of a word in a given context is a function of the distinctions it is supposed to convey in that context. According to this view, there may be no overlap between uses of the same word across contexts. Psychologists who have observed contextual effects on encoding have often reached a similar conclusion (e.g., Jacoby, Craik, $\&$ Begg, 1979). In the terms of this paper, this approach argues that all of a concept's properties are CD. The proposal that some of a concept's properties are CI and others are $\mathrm{CD}$ lies between these two theories of meaning. Consequently, evidence for the existence of $\mathrm{CI}$ and $\mathrm{CD}$ properties in concepts would have implications for theories of natural language semantics. ${ }^{1}$

The definitions of $\mathrm{CI}$ and $\mathrm{CD}$ properties lead to several empirical predictions. First, in a given context, all CI properties should be available and irrelevant CD properties should not. This is because CI properties are always activated by their respective words, whereas irrelevant $C D$ properties remain inactive due to lack of contextual activation. The second prediction follows from the definition of CI properties. Since CI properties are always activated by the respective words on all occasions, they should be unaffected by contextual relevance: A CI property should be just as available in an irrelevant context as in a relevant context. This assumes that the activation of a CI property by a word maximally activates that property. The third prediction follows from the definition of $C D$ properties. Since CD properties depend on relevant contexts for activation, they should be available in working memory for processing when the context is relevant and unavailable when the context is irrelevant. The alternative hypotheses are (1) all the properties in a concept are activated by the respective word on all occasions and (2) all the properties active in a concept are determined by context.

These hypotheses and their alternatives are contrasted in the two experiments that follow. The first experiment tests these predictions in the propertyverification task. The second experiment tests these predictions in judgments of similarity. Evidence from other current work is also brought to bear on these issues. Finally, the relevance of CI and CD properties to crossclassification, problem solving, metaphor and sentence comprehension, and the semantic-episodic distinction is discussed.

\section{EXPERIMENT 1}

A version of the property-verification task was used to test the predictions following from the definitions of $\mathrm{CI}$ and $\mathrm{CD}$ properties. On each trial, subjects read a sentence containing an underlined subject noun. Several seconds later, the label for a property was presented, and subjects indicated whether or not the subject noun in the preceding sentence possessed the property.

The logic of the experiment is as follows. If the property for a trial is CI information of the subject noun, 
then verification time should not vary across sentence contexts. In particular, verification time should be no less when the sentence context is related to the property than when the sentence context is unrelated. This is because the property, being CI, is always activated by the subject noun itself and therefore is not dependent on context for activation. However, if the property for a trial is $C D$ information of the subject noun, then verification time should depend on context. Specifically, verification time should be much less when the sentence context is related to the property than when the sentence context is unrelated. This is because the property, being $\mathrm{CD}$, is not activated by the subject noun and therefore is dependent on context for activation. If the difference between related and unrelated contexts for CD properties is substantial (i.e., on the order of several hundred milliseconds), this would suggest that $C D$ properties are, in fact, inactive in irrelevant contexts.

\section{Method}

Procedure. Subjects looked into a modified Siliconix tachistoscope and rested their forefingers on two response buttons $7 \mathrm{~cm}$ apart. When prepared for a trial, subjects pressed the "start" button (positioned colinearly and midway between the two response buttons) with the same finger used to press the "true" button. After a 500-msec interval, a context sentence appeared in the top field of the tachistoscope. All sentences began with "The," followed by an underlined subject noun and a predication of the subject noun. Subjects were instructed to fully comprehend the sentence and to read it out loud. The context sentence was removed after $6 \mathrm{sec}$, and a property label immediately appeared in the bottom field; subjects did not read the label aloud. If the subject noun in the context sentence possessed the property, subjects pressed the "true" response button; otherwise, they pressed the "false" response button. For each trial, time was measured from the onset of the property to the point at which a response was detected. Subjects were instructed to respond as quickly as possible, but to avoid making errors.
Subjects received 24 practice trials and 60 test trials. There was a short break between the practice and test trials. Subjects could take a break anytime during the test trials, but they rarely did. Following the last test trial, subjects were asked a series of questions concerning their strategies.

Subjects and Materials. The subjects were 19 Stanford students participating for pay or course credit. One subject's data were not used because of an error rate exceeding $15 \%$ (the average error rate for the remaining subjects was $2.8 \%$ ). The materials consisted of context sentences and properties, related as discussed next. Examples of the materials are shown in Table 1.

Trues. Thirty properties were chosen for the "true" trials; 15 were randomly assigned to the $\mathrm{Cl}$ condition and 15 to the CD condition. The average number of syllables per property did not vary between conditions $[4.06$ and 4.13 for the CI and CD properties, respectively; $t(28)=.14, p>.30$ ]. For each $\mathrm{CI}$ property, three context sentences were constructed. Two of these contained the same subject noun, which was highly related to the property; the predicate for one of these sentences was related to the property (the related-context sentence), and the predicate for the other was unrelated (the unrelated-context sentence). Degree of relatedness was determined by the ratings of an independent group of subjects, as reported later.) The remaining context sentence contained a subject noun, weakly related to the property, and an unrelated predicate; this sentence served as a control sentence (to be explained in a moment).

Similarly, for each of the $15 \mathrm{CD}$ properties, three context sentences were constructed. Two of these contained the same subject noun, which was weakly related to the property; the predicate for one of these sentences was related to the property (the related-context sentence), and the predicate for the other was unrelated (the unrelated-context sentence). The remaining sentence (the control sentence) contained a subject noun, highly related to the property, and an unrelated predicate.

A control sentence in the $\mathrm{CI}$ condition (having a weakly related subject noun and an unrelated predicate) served as a contrast to verify that the other two context sentences for the property had a subject noun highly related to the property. The time to verify the unrelated-context sentence (having a highly related subject noun and an unrelated predicate) should be less than that for the control sentence. Similarly, a control sentence in the $\mathrm{CD}$ condition (having a highly related subject noun and an unre-

Table 1

Examples of Materials Used in Experiment 1

\begin{tabular}{|c|c|c|}
\hline Property & Context & Item \\
\hline Has a smell & $\begin{array}{l}\text { Unrelated } \\
\text { Related } \\
\text { Control }\end{array}$ & $\begin{array}{l}\text { Context-Independent "True" Items } \\
\text { The skunk was under a large willow. } \\
\text { The skunk stunk up the entire neighborhood. } \\
\text { The fire was easily visible through the trees. }\end{array}$ \\
\hline Can contain money & $\begin{array}{l}\text { Unrelated } \\
\text { Related } \\
\text { Control }\end{array}$ & $\begin{array}{l}\text { The bank had been built ten years ago. } \\
\text { The bank was robbed by three bandits. } \\
\text { The jar was an old antique. }\end{array}$ \\
\hline Can be walked upon & $\begin{array}{l}\text { Unrelated } \\
\text { Related } \\
\text { Control }\end{array}$ & $\begin{array}{l}\text { Context-Dependent "True" Items } \\
\text { The roof had been renovated prior to the rainy season. } \\
\text { The roof creaked under the weight of the repairman. } \\
\text { The tightrope was high off the ground. }\end{array}$ \\
\hline \multirow[t]{2}{*}{ Where cooking can occur } & $\begin{array}{l}\text { Unrelated } \\
\text { Related } \\
\text { Control }\end{array}$ & $\begin{array}{l}\text { The hospital was internationally famous for its progressive techniques. } \\
\text { The hospital was quiet when dinner was served. } \\
\text { The kitchen had been repainted over the holidays. }\end{array}$ \\
\hline & & "False" Items \\
\hline Has gills & & The cheese was growing moldy in the refrigerator. \\
\hline Can be tied in a knot & & The refrigerator was set to a low temperature to cool the beer. \\
\hline
\end{tabular}


Table 2

Average Association-to-Property Ratings for Experiment 1 Materials

\begin{tabular}{|c|c|c|c|c|c|}
\hline \multirow[b]{3}{*}{ Condition } & \multicolumn{5}{|c|}{ A Priori Relatedness to Property } \\
\hline & \multicolumn{2}{|c|}{ Subject Nouns } & \multicolumn{3}{|c|}{ Predicates } \\
\hline & Noncontrol* & Control & Control** & Unrelated & Related \\
\hline Context-Independent & 6.80 & 3.30 & 3.00 & 3.10 & 6.60 \\
\hline Context-Dependent & 3.18 & 6.72 & 3.22 & 2.68 & 6.25 \\
\hline
\end{tabular}

*These are the subject nouns for the unrelated and related context sentences. **Unrelated.

lated predicate) served as a contrast to verify that the other two context sentences for the property had a subject noun weakly related to the property. The time to verify the unrelated context sentence (having a weakly related subject noun and an unrelated predicate) should be longer than that for the control sentence.

Ratings were obtained to confirm the assumed relations between the subject nouns and properties, and between the predicates and properties. Of primary importance is that context be manipulated equally for the $C I$ and $C D$ conditions. This insures that an effect of context on CD subject nouns but not on CI subject nouns for the latency data cannot be attributed to CD materials having more relatedness for related predicates or less relatedness for unrelated predicates than the CI materials. Four judges rated the 60 subject nouns first (30 for the control and 30 for the noncontrol sentences) and the 90 predicates second ( 3 for each property). Subjects read either a subject noun or a predicate on one side of an index card and then flipped the card to read the property. Subjects rated how much the subject noun or predicate made them think of the property. Subjects used a scale from 1 to 7 , on which 1 meant the property did not come to mind at all and 7 meant the property immediately came to mind. Within each group, the cards were randomly ordered for each subject.

An ANOVA was performed on the ratings for the subject nouns. The two factors of interest were condition (i.e., CI vs. CD) and relatedness (i.e., weakly vs. highly related). Note that for the CI materials, the nouns in the related- and unrelatedcontext sentences were supposed to be highly related and the nouns in the control sentences were supposed to be weakly related to their respective properties. For the $\mathrm{CD}$ materials, the nouns in the related-and unrelated-context sentences were supposed to be weakly related and the nouns in the control sentences were supposed to be highly related to their respective properties. The mean ratings from this analysis are shown in Table 2. There was no effect of CI/CD $[F(1,3)=1.74, p>.25]$ there was an effect of relatedness $[F(1,3)=204.54, p<.001]$, and there was no interaction between these two factors $(F<1)$ Thus the assumed difference in relatedness was substantial and equivalent for the $\mathrm{CI}$ and $\mathrm{CD}$ materials. A similar ANOVA was performed for the predicates. Again, there was no effect of $\mathrm{CI} / \mathrm{CD}[\mathrm{F}(1,3)=1.71, \mathrm{p}>.25]$ and no interaction of this factor with relatedness $[F(1,6)=1.19, p>.25]$. The predicates for the control and unrelated-context sentences did not differ in relatedness $(F<1)$. However, the predicates for the related-context sentences were higher in relatedness than those for the unrelatedcontext sentences $[F(1,6)=89.97, p<.001]$ and those for the control sentences $[F(1,6)=79.28, p<.001]$. Thus the assumed difference in relatedness was again substantial and equivalent for the $\mathrm{CI}$ and $\mathrm{CD}$ materials. Crucial to the interpretation of the latency results are the findings that (1) the related predicates for the CD materials were not higher in relatedness than those for the $C I$ materials $(F<1)$ and $(2)$ the unrelated predicates for the $C D$ materials were not lower in relatedness than those for the $\mathrm{CI}$ materials $(\mathrm{F}<1)$.

Falses. Thirty context sentence/property pairs were constructed, each context sentence having a subject noun that clearly did not possess the property. The context sentences and properties used were similar in nature to those for the "true" materials. Five of the 30 "true" context sentences presented to a subject (as discussed in the Design section) contained a subject noun and a predicate both highly related to the same property (i.e., the CI related-context sentences). Therefore, 5 of the 30 "false" context sentences also contained a subject noun and a predicate both highly related to some property; however, this was not the property actually tested (i.e., for the "false" items, the subject noun could not possess the property). Creating some "false" items in this manner made it impossible for subjects to discriminate the "true" from the "false" items on the basis of subject-predicate-property relations.

Practice items. Twenty-four context sentence/property pairs were constructed; half were true and half were false. These items were similar in nature to the "true" and "false" test items. Also, the distribution of item types was similar to that found in the set of test items.

Design. Three lists were constructed. Each contained the same 30 context sentence/property pairs for the "false" items and the same 30 properties for the "true" items. The lists differed only with respect to the context sentences for the "true" properties, as discussed next.

The 15 CI properties for the "true" items were randomly divided into three groups of five properties each; the $15 \mathrm{CD}$ properties were also randomly divided into three groups of five properties each. The 30 "true" context sentences in a given list consisted of (1) the control sentences from one CI group and one CD group, (2) the unrelated-context sentences from a second CI group and a second CD group, and (3) the relatedcontext sentences from the remaining $\mathrm{CI}$ group and the remaining CD group. Each of the three context sentence types for each property group was instantiated in one and only one of the lists. This rotation of context sentence type through property group and list was done as randomly as possible, given the necessary constraints of a Latin square.

The 24 practice items were presented in the same random order to all subjects. The 60 test items were presented to each subject in a different, computer-generated, random order. Half the subjects used their right forefingers to press the "start" and "true" buttons and their left forefingers to press the "false" button; the other subjects had the inverse assignment. Subjects were assigned randomly to one of the six lists by hand assignment cells of the design, three subjects per cell.

\section{Results}

Latencies for the correct true trials were analyzed as follows. Averages were computed separately across subjects and across items (i.e., properties). For each subject, the average latency was determined for each of the six subject relation by predicate relation conditions. For each property in the $\mathrm{CI}$ and $\mathrm{CD}$ conditions, the average latency was determined for each of the three predicate relations. The results for the subject averages are shown in Table 3. Separate subject relation by predicate rela- 
Table 3

Average Latencies and Error Rates per Subject for Correct True Trials (Experiment 1)

\begin{tabular}{|c|c|c|c|c|c|c|}
\hline \multirow[b]{3}{*}{ Condition } & \multicolumn{6}{|c|}{ Predicate Relation } \\
\hline & \multicolumn{2}{|c|}{$\begin{array}{c}\text { Control } \\
\text { (Unrelated) }\end{array}$} & \multicolumn{2}{|c|}{ Unrelated } & \multicolumn{2}{|c|}{ Related } \\
\hline & $\mathbf{L}$ & $\% \mathrm{E}$ & $\mathrm{L}$ & $\% \mathrm{E}$ & $\mathrm{L}$ & $\% \mathrm{E}$ \\
\hline Context-Independent & 1335 & 11 & 1113 & 0 & 1145 & 3 \\
\hline Context-Dependent & 1098 & 1 & 1404 & 11 & 1259 & 3 \\
\hline
\end{tabular}

Note $-L=$ average latency; $\% E=$ error rate.

tion ANOVAs were performed on the subject averages and item averages. The results of both analyses were combined to compute min $\mathrm{F}^{\prime}$ planned comparisons of interest (H. H. Clark, 1973).

For the CI items, the control sentences led to longer latencies than the unrelated-context sentences $\left[\min F^{\prime}(1,89)=15.80, p<.001\right]$. For the CD items, the control sentences led to shorter latencies than the unrelated-context sentences $\left[\min \mathrm{F}^{\prime}(1,90)=24.80\right.$, $p<.001]$. These two results show that (1) the subject nouns in the $\mathrm{CI}$ noncontrol sentences were in fact highly related to their respective $\mathrm{CI}$ properties, and (2) the sub. ject nouns in the CD noncontrol sentences were in fact weakly related to their respective properties. ${ }^{2}$

The remaining results pertain only to the noncontrol sentences. For the $\mathrm{CI}$ items, there was no difference between related- and unrelated-context sentences (min $F^{\prime}<1$; the subject's $F$ and item's $F$ were also less than 1). For the $C D$ items, related-context sentences led to shorter latencies than unrelated-context sentences $\left[\min F^{\prime}(1,90)=5.97, p<.025\right]$. For the unrelatedcontext sentences, the latencies were less for the $\mathrm{CI}$ items than for the CD items $\left[\min \mathrm{F}^{\prime}(1,90)=22.13\right.$, $\mathrm{p}<.001]$. For the related-context sentences, there was a marginal difference between the $\mathrm{CI}$ and $\mathrm{CD}$ items $\left[\min F^{\prime}(1,89)=3.16, .10>p>.05\right] ;$ however, the subject's $F$ was significant $[F(1,34)=9.66, p<.01]$, as was the item's $F[F(1,56)=4.70, p<.05]$. There was a significant Subject Relation by Predicate Relation interaction for the noncontrol sentences $\left[\min F^{\prime}(1,90)=4.19, \mathrm{p}<.05\right]$.

These data indicate that context had no effect on the CI items but had an effect on the CD items. More specifically, related contexts did not increase the priming of properties when the subject noun was highly related to the target property. However, related contexts did increase the priming of properties when the subject noun was weakly related to the property. It is not clear whether the facilitation caused by related contexts for the $\mathrm{CD}$ subject nouns was equivalent to the facilitation caused by the CI subject nouns themselves.

Mean latency for the correct "true" trials was $1,226 \mathrm{msec}$, and for the correct "false" trials, it was $1,253 \mathrm{msec}$. The average "true" latency for 13 of the 18 subjects was less than the average "false" latency. The average error rate per subject for all 60 test trials was $2.8 \%$. The average error rate per subject for the 30 "true" trials was 5\%, and for the 30 "false" trials, it was $.6 \%$. The average error rates per subject for the six subject relation by predicate relation cells of the design are shown in Table 3. These data, in conjunction with the latencies, indicate there was no speed-accuracy tradeoff. Notably, the most errors occurred for sentences having weakly related subject nouns and unrelated contexts. When questioned at the end of the experiment about errors on these sentences, subjects said they believed the correct response was "true" in all cases. They also indicated they had realized this almost immediately after responding "false." This suggests that $\mathrm{CD}$ properties in irrelevant contexts are normally inactive and that errors for these sentences occur when subjects decide to respond prior to this information's becoming active.

During the postexperimental interviews, most subjects reported not trying to guess properties before their presentation. The most common strategy involved focusing attention on the subject noun and forgetting the predicate while waiting for the property. All subjects reported either rehearsing the subject noun or focusing on it during the waiting period. Several subjects said they imaged referents of the subject nouns; several said they rehearsed the subject nouns once and then focused on them until presentation of the property. All but one subject reported that the psychological status of the predicate was either peripheral or gone from consciousness. Some subjects indicated that trying to maintain the predicate interfered with the task. In general, subjects appeared to be focusing only on the subject noun, believing this would maximize their ability to perform the verification task. Interestingly, the predicates still had an effect, as shown by the results for the CD properties.

\section{Discussion}

These data are consistent with the distinction between $\mathrm{CI}$ and $\mathrm{CD}$ properties. Some properties are CI because their verification is unaffected by contextual relevance. Others are $C D$ because their verifications are faster in relevant contexts than in irrelevant contexts (a facilitation of $145 \mathrm{msec}$ in this experiment). These data suggest that CD properties are not activated in irrelevant contexts. Specifically, properties weakly related to subject nouns were verified 237 and $291 \mathrm{msec}$ more slowly than properties highly related to subject nouns for control and unrelated sentences, respectively. It seems unlikely that differences of this size could occur if the weakly related properties were activated by their respective words. Rather, these differences may largely reflect the time it takes to activate these properties.

These results provide a functional account of property availability: Highly accessible properties of a concept are available independent of context, whereas less 
accessible ones are available only in relevant contexts. Conrad (1978) has also found results consistent with this account. Her task employed interference in a colornaming task as the dependent variable. On each trial, subjects read a sentence and reported the ink color of a subsequent word. For "true" trials, the word in colored ink was either a highly related or weakly related property of the last word in the sentence. This factor was crossed with whether or not the sentence context made the property in colored ink relevant to the last word in the sentence. The results were analogous to those in this experiment. The amount of interference for the highly related properties was independent of contextual relevance. For the weakly related properties, however, interference occurred only when the context made the critical property relevant to the final sentence word.

Tabossi and Johnson-Laird (1980) also found results similar to those reported here. In a property-verification task, in which only predicate relatedness was systematically varied, subjects were faster to verify properties in relevant than in irrelevant contexts. This indicates that some of the properties must have been CD. If they had all been $\mathrm{CI}$, this effect would not have occurred, given the results of the current experiment and those of Conrad (1978). Besides using contexts that primed the target property and contexts that primed no property of the target noun, Tabossi and Johnson-Laird also used contexts that primed a property of the target noun other than the target property. This third type of context led to the longest verification times. But since strength of association between the target noun and the target property was not controlled, it is not clear whether this interference effect occurred for CI properties, CD properties, or both. Nevertheless, this effect further constrains a functional account of property availability: Contexts can inhibit the activation of properties, although this may not be true of all properties.

In the current experiment and in Tabossi and Johnson-Laird's (1980) Experiment 2, the context sentences were presented $6 \mathrm{sec}$ before the target properties (Conrad, 1978, did not report the details of her procedure). These experiments, therefore, are not informative at any level more specific than a functional one. This is because both automatic and conscious priming effects have been shown to occur well within $2 \mathrm{sec}$ (Neely, 1977; Posner \& Snyder, 1975; Swinney, 1979; Tanenhaus et al., 1979). However, Posner and Snyder's (1975) theory of attention may be an interesting way to think about property availability. They propose two types of attentional processes: (1) unconscious, automatic processes that are the result of past learning, and (2) conscious processes that are subject to capacity limitations. Although both types cause priming, conscious processes do so more slowly and interfere with other processing. Viewing property availability in this framework, the perception of a word may automatically activate its $\mathrm{CI}$ properties. In contrast, conscious attention may be responsible for activating relevant $C D$ properties and for keeping both types of property active via rehearsal. (It is also possible that some $C D$ properties are automatically activated.) Finally, focusing conscious attention away from automatically activated properties may eventually inhibit their verification.

This information processing account of property availability is consistent with the results reported here and with those of Conrad (1978): CI properties are always available because they are automatically activated by their respective words and are kept active by conscious rehearsal; $C D$ properties are available only in irrelevant contexts because they are either automatically or consciously activated via contexts and are kept active by conscious rehearsal. This account also explains the Tabossi and Johnson-Laird (1980) interference effect: The verification of an automatically activated property may be inhibited if context focuses attention away from it.

Priming in this experiment appears at first glance to be nonadditive (cf. Foss, Cirilo, \& Blank, 1979). For the CI properties, priming from the word and from the context did not add, since CI related-context sentences did not lead to faster verifications than CI unrelatedcontext sentences (i.e., there was no additional priming from the contexts). But in the Posner and Snyder (1975) framework, this pattern could well be additive. CI properties may receive their initial activation from encoding their respective words, this automatic activation dissipating within a few hundred milliseconds. But once these properties become active, they may receive conscious attention, which increases as automatic activation decreases. The activation of $\mathrm{CI}$ properties may therefore be additive in the sense that different processes are responsible for maintaining a high level of activation.

Finally, it is necessary to comment on the activation of $\mathrm{CD}$ properties. Functionally speaking, these properties are available in relevant but not in irrelevant contexts. But trying to explain this in information processing terms quickly becomes complex. CD properties may become available in two ways. First, they may actually be stored in a concept and be activated by contexts containing similar or associated information. Certain nounproperty relations in this experiment appear to have been of this type (e.g., "snake-can be a pet"; "fingerscan be used for eating"; "frog-can be eaten"). Just how contexts activate these properties is a topic worthy of future interest. The second way $C D$ properties can become available is via inference. Certain CD properties may not be stored in a concept but may be computed with various inference procedures (e.g., cognitive economy; Collins \& Quillian, 1969; Conrad, 1972). Certain noun-property relations in this experiment may have been of the inference type for certain subjects (e.g., "basketball-can float"; "pencil-can pierce something"; "zebra-has ears"). The range and nature of these inference processes are other topics worthy of future interest. 
In particular, they appear to present a problem for theories of semantics, which usually try to characterize word meanings with finite sets of properties.

\section{EXPERIMENT 2}

A much different task was used in this experiment to further demonstrate the distinction between $\mathrm{CI}$ and $\mathrm{CD}$ information. Subjects judged the similarity of instance pairs drawn from various categories (e.g., "desk-sofa" from "furniture"). Two types of categories, common and ad hoc, were used. Common categories are highly conventional categories, such as those studied by Rosch, Smith, and their colleagues (e.g., Rips, Shoben, \& Smith, 1973; Rosch, 1975; Rosch \& Mervis, 1975; Rosch, Mervis, Gray, Johnson, \& Boyes-Braem, 1976; Smith et al., 1974). Examples of these categories are "birds," "furniture," and "vegetables." In contrast, ad hoc categories are highly unusual categories that are rarely, if ever, used (Barsalou, Note 2). As a result, they are not well established in memory. Examples of these categories are "things that have a smell," "things that float," and "things that can be thrown."

Half the subjects received the category name prior to judging the similarity of each pair (the context condition); the remaining subjects did not receive the category names (the no-context condition). The predictions for this experiment are derived from Barsalou's (Note 2) finding that the properties shared by common category instances are usually $\mathrm{CI}$, whereas the properties shared by ad hoc category instances may often be CD. For example, it is fairly obvious that carrots and broccoli share properties common to vegetables. However, it is not obvious that basketballs and logs share properties common to things that float. It follows that the similarity of pairs from ad hoc categories should be greater when these pairs are preceded by their category names than when they are not. This is because the category names activate shared properties that are normally inactive. Thus, there should be more common properties active in the context than in the no-context condition for ad hoc categories. In contrast, the similarity of pairs from common categories should not be increased by the addition of category names. This is because the shared properties are equally active with and without context. Combining the different patterns for ad hoc and common categories, the central prediction for this experiment is that there should be a Context by Category Type interaction. The difference in similarity between pairs from common and ad hoc categories should be less with context than without.

One other prediction for this experiment also follows from Barsalou (Note 2). The similarities should generally be greater for common than for ad hoc categories. This is because common categories are some of the categories having the highest intraclass similarity, whereas the exemplars of ad hoc categories often have much less in common. This effect is not relevant to the purpose of the experiment, but it is expected to occur.

\section{Method}

Materials and Design. Twenty common categories were selected from Battig and Montague (1969) and Rosch (1975). These categories intuitively appeared to be well-known and often used. Twenty ad hoc categories were selected that appeared to be atypical and infrequently used. Two instances were chosen from each category. The common category instances were selected such that the category properties shared by these instances appeared to be CI. The ad hoc category instances were selected such that the category properties shared by these instances appeared to be $\mathrm{CD}$. Examples of the materials are shown in Table 4.

Eight judges verified that the $\mathrm{Cl}$ properties were indeed more accessible for the common category instances than the CD properties were for the ad hoc category instances. The judges read the name of an instance on one side of an index card and then read the category name on the other side. They rated how much reading the instance name brought to mind the properties associated with the category name. The judges used a scale from 1 to 7 , on which 1 meant the properties did not come to mind at all and 7 meant the properties immediately came to mind. Each judge rated only one instance per pair, to avoid priming effects between instances. So, four judges rated each instance, and each judge rated 20 common category instances and 20 ad hoc category instances. The 40 cards were randomly ordered for each judge. The mean accessibility rating for common category instances was 5.52, and for ad hoc category instances, it was $2.32[\mathrm{~F}(1,6)=164.39, \mathrm{p}<.001]$. This indicates that there was a substantial difference in property accessibility between the common and ad hoc category materials.

Two versions of the pairs were constructed. In each version, the 40 pairs were randomly ordered, as were the two words in each pair. The pairs were typed onto two pages, 20 per page. In the context condition, the category name appeared to the left of each pair; in the no-context condition, the pairs appeared in isolation. Thus, there were four lists: two versions of the context list and two versions of the no-context list. To the right of each pair appeared the integers from 1 to 9 . At the top of the page, above this block of response scales, appeared labels for the scale. Above 1 appeared "not similar at all," above 9 appeared "very similar," and above the remaining integers appeared "increasing similarity."

Subjects and Procedure. The subjects were 28 Stanford students participating to earn course credit. Fourteen subjects were randomly assigned to the context condition and 14 to the nocontext condition. Within each of these groups, half the subjects received each version of the list. Subjects were asked to think of the thing to which each word in a pair referred. They were then

Table 4

Examples of Materials Used in Experiment 2

\begin{tabular}{ll}
\multicolumn{1}{c}{ Category } & \multicolumn{1}{c}{ Pair } \\
\hline $\begin{array}{l}\text { birds } \\
\text { furniture } \\
\text { kitchen utensils } \\
\text { beverages }\end{array}$ & $\begin{array}{l}\text { Common Categories } \\
\text { robineagle } \\
\text { sofa-desk } \\
\text { cup-plate } \\
\text { coffee-milk }\end{array}$ \\
$\begin{array}{ll}\text { plunder taken by conquerors } \\
\text { possible gifts } \\
\text { taken on camping trips }\end{array}$ & $\begin{array}{l}\text { slaves-jeweiry } \\
\text { can be a pet }\end{array}$ \\
\hline
\end{tabular}


Table 5

Effects of Context and Category Type on Average Similarity (Experiment 2)

\begin{tabular}{ccc}
\hline & \multicolumn{2}{c}{ Category Type } \\
\cline { 2 - 3 } Conditions & Ad hoc & Common \\
\hline Context & 3.67 & 5.28 \\
No Context & 2.52 & 5.73 \\
\hline
\end{tabular}

to judge the similarity of these two referents. Subjects were told about the scale and asked to circle one of the numbers for each pair to indicate their judgment. Subjects in the context condition were told that each pair was preceded by the name of a category to which the words in the pair belonged.

\section{Results}

The reliability of the mean ratings for the pairs was computed using the intraclass correlation for averages (Guilford \& Fruchter, 1973). The reliability of the mean ratings was .96 for the no-context condition and .88 for the context condition.

A four-way ANOVA, context by category type by categories by subjects, was performed on the data. Since categories and subjects were both random factors, it was necessary to compute quasi-F's (H. H. Clark, 1973; Winer, 1971). The relevant means from this analysis are shown in Table 5. There was no main effect of context $\left(\mathrm{F}^{\prime}<1\right)$. However, there was a main effect of category type $\left[\mathrm{F}^{\prime}(1,62)=52.80, \mathrm{p}<.001\right]$, common categories exhibiting more similarity than ad hoc categories, as predicted. Most important, there was a Context by Category Type interaction $\left[F^{\prime}(1,48)=12.50, p<.001\right]$. The difference between common and ad hoc categories was less with context than without, as predicted by the definitions of $\mathrm{Cl}$ and $\mathrm{CD}$ properties. Planned comparisons were computed by performing separate ANOVAs on only the relevant data. For the ad hoc categories, the similarities were higher with context than without $\left[F^{\prime}(1,35)=8.31, p<.01\right]$. In contrast, the context manipulation had no effect on the similarity of common category pairs $\left(F^{\prime}<1\right)$.

\section{Discussion}

The presence of context reduced the difference in similarity between common and ad hoc categories by one-half. Without context, the difference was 3.21 , whereas with context, the difference was 1.61 . This is further support for the existence of $\mathrm{Cl}$ and $\mathrm{CD}$ information. The category properties shared by ad hoc category instances were $C D$, since the similarity of these pairs was greater with relevant context than without. Relevant context was necessary to activate shared properties not activated by the words themselves. In contrast, the category properties shared by common category instances were $\mathrm{CI}$, since the similarity of these pairs did not change across context. Relevant context was not required to activate shared properties activated by the words themselves.

Again, these results only provide support for a func- tional account of property availability: Some properties of a concept are available independent of context, since they are activated by the respective word, whereas others become available only in relevant contexts. Since subjects had as much time as they needed to perform their judgments, it was not possible to observe the time course of property activation. For this reason, it is not possible to test explanations based on the concepts of automatic and conscious attentional processes. However, the application of the Posner and Snyder (1975) framework to property availability, as discussed for Experiment 1 , also makes sense in the context of the current experiment.

\section{GENERAL DISCUSSION}

These experiments demonstrate the existence of $\mathrm{CI}$ and $\mathrm{CD}$ properties. $\mathrm{CI}$ properties were shown to be activated by their respective words independently of context. In Experiment 1, the verification of CI properties was unaffected by the relevance of sentence frames. In Experiment 2, the similarity of two concepts was not increased when a context relevant to shared $\mathrm{CI}$ properties was presented. In contrast, $C D$ properties were shown not to be activated by their respective words, but only by relevant contexts in which the words appeared. In Experiment 1, the verification of $C D$ properties was faster in relevant than in irrelevant contexts. In Experiment 2, the similarity of two concepts increased when a relevant context activated shared CD properties.

These findings have implications for theories of natural language semantics and for semantic memory models. Given the existence of CD properties, the meaning of a word is not a fixed set of properties that is activated as a whole every time the respective word is encoded. Rather, the meaning of a word also contains weakly associated and inferable properties that are inactive in irrelevant contexts and active in relevant contexts. Given the existence of CI properties, the meaning of a word is not completely determined by context. Rather, certain properties appear to be automatically activated by a word independently of context. These findings indicate that accounts of natural language semantics should include assumptions regarding (1) the accessibility of semantic properties and (2) the impact of context on the accessibility of these properties.

The remainder of this paper addresses the roles of $\mathrm{CI}$ and $\mathrm{CD}$ properties in the following cognitive phenomena: cross-classification, problem solving, metaphor, and sentence comprehension. Also discussed are implications for the semanticepisodic distinction.

\section{Cross-Classification}

Any concept is potentially cross-classifiable into an indefinitely large number of categories (see Barsalou, Note 2). For example, "chair" belongs to "furniture," "gifts," "things to sell at a garage sale," "things that can 
be used to hold a door open," and so on. Some of the classifications of a concept may be explicitly represented in memory (e.g., a robin is a bird) such that they can be directly accessed from the word for the concept. Many cross-classifications, however, may be implicit, in that they are not prestored but are computed by various inference processes when necessary (cf. Camp, Lachman, $\&$ Lachman, 1980). For example, there could be a process that takes any property, $\mathrm{X}$, associated with a concept and infers that instances of the concept belong to the category of things that exhibit X. "Bear" can be cross-classified into things that have fur because fur is associated with bears.

Barsalou (Note 2) proposed a model of how implicit cross-classifications are computed. The model's first assumption is that for each possible classification, there is a set of criterial properties (coupled with a decision rule) used to discriminate category instances. The model's second assumption is that the properties active for a concept on a given occasion are a subset of the properties in that concept, this subset containing $\mathrm{CI}$ and CD properties. It follows that the implicit crossclassifications of a concept computable in a given context are those whose criterial properties are contained in the concept's active subset. Consequently, crossclassifications based on CI properties should be possible on any occasion. The category "things that are round" should be computable on all occasions for "basket. ball" if "round" is CI for "basketball." Crossclassifications based on CD properties should similarly be possible on occasions when these properties are active, but they should not be possible when these properties are inactive. "Things that float" should only be computable for "basketball" if a relevant context (e.g., a need for a life preserver) activates "floats" in the concept for "basketball." Barsalou (Note 2) reports data consistent with this view of cross-classification.

\section{Problem Solving}

The account of cross-classification just discussed bears upon functional fixedness in problem solving (Duncker, 1945). Functional fixedness is the phenomenon of an object's typical function preventing insight into other, less typical functions that might be more useful in a particular situation. In one problem, subjects are presented several objects and asked to use them to support a board (Duncker, 1945). Crucial to solving this problem is using a pair of pliers as a support. But since "can provide support" is not a salient function of pliers, subjects often have difficulty solving the problem. In many such cases, the salient function may be CI, whereas the less salient function may be $\mathrm{CD}$. Perception of the less salient cross-classification may depend on attending to the critical object in the appropriate mental context such that the CD-based classification can be inferred. For example, the CI properties in the concept for things that could support the board might activate relevant $\mathrm{CD}$ properties in the representation of pliers if these two concepts were simultaneously active in memory. These CD properties could then be used to infer that "pliers" belongs to "things that could support the board."

Finding a solution to this problem may be delayed because subjects are misled by the CI properties of the critical object. For example, "to grasp something" is probably CI for "pliers." Once this property is automatically activated, subjects may rule out pliers as a possible support. Consequently, the object is not attended to in the context necessary for activating the relevant properties. When subjects get desperate, this initial classification may be discarded such that the object is more carefully scrutinized and properly classified. In support of this, Duncker (1945) and Glucksberg and Danks (1968) have found that it takes longer to solve a problem when attention is drawn to the interfering CI properties. Duncker (1945) distracted subjects by having them use the pliers to grasp something before solving the problem. Glucksberg and Danks (1968) either mentioned the word for the critical object or labeled the object with a nonsense syllable. Mentioning the word delayed solutions, presumably because hearing the word automatically activated interfering CI properties.

Sometimes the activation of $\mathrm{CI}$ properties may facilitate finding a solution. In the candle problem, subjects are given a candle, some matches, and a box of tacks; their task is to attach the candle to the wall and light it (Duncker, 1945). Usually it takes subjects a while to cross-classify the box as something that could contain the candle. However, Glucksberg and Weisberg (1966) found that having the experimenter label the box as "box" resulted in faster solutions than when the box of tacks was simply labeled "tacks." They argued that using "box" drew attention to an object that was otherwise obscured by what it contained. However, another factor may be involved as well. Assuming that "contains things" is CI for "box," it follows that this property should become available when subjects hear the experimenter say "box." Having this property available should then facilitate cross-classifying the box as something that could contain the candle.

\section{Metaphor and Sentence Comprehension}

Ortony (1979) has proposed that metaphoricity depends on a particular type of salience imbalance. Specifically, the property brought to mind by a metaphor should have low salience for the subject and high salience for the referent of the metaphor. For "sermons are sleeping pills," the property "induces drowsiness or sleep" has low salience for "sermons," but high salience for "sleeping pills."

In many metaphors, the shared property may be $\mathrm{CD}$ in the subject and $\mathrm{CI}$ in the referent. In these cases, the CI property in the referent may automatically activate the corresponding CD property in the subject (see Glucksberg, Dial, \& Bookin, Note 3). That is, the referent serves as context for the subject, activating relevant 
CD properties. It follows from Ortony's (1979) analysis that the best metaphors should be those in which the shared property is $\mathrm{CD}$ in the subject and $\mathrm{CI}$ in the referent. This is because these are the cases in which salience imbalance is maximized. Metaphors in which the shared property is not $\mathrm{CD}$ in the subject should not appear as metaphorical, since the property may come to mind for the subject outside the context of the referent.

In general, the mechanism of $\mathrm{Cl}$ properties in one word activating $\mathrm{CD}$ properties in other words may be central to sentence comprehension. As shown in Experiment 1 , the predicate in a sentence can bring to mind properties of the subject (e.g., the predicate in "The rag was used to start the fire" brings to mind "is flammable" for "rag"). There appear to be many other ways in which CI properties of one sentence word activate CD properties of another sentence word. For example, CI properties can bring to mind the appropriate senses of ambiguous words. ${ }^{3}$ This occurs from direct objects to verbs. For "John ate X," the instantiation of X determines the sense of "eat" that comes to mind (e.g., consider $X=$ soup, a sandwich, a steak, and so on). Similarly, the CI properties of a noun serve to disambiguate modifiers. For "the broken X," the instantiation of X determines the sense of "broken" that comes to mind (e.g., consider $\mathrm{X}=$ bowl, truck, plan, and so on). Similarly, the CI properties of an object in a prepositional phrase determine the sense of the preposition that comes to mind. For "on the X," the instantiation of X determines the sense of "on" that comes to mind [e.g., consider $\mathrm{X}=$ table, television, roof (where the subject is a person vs. a fly), and so on]. In general, converging on the intended meaning of a sentence may often involve selecting the properties associated with individual words that result in the most coordinated interpretation. This selection mechanism can be characterized, at least to some extent, as the activation of relevant $C D$ properties in some words by CI properties in other words. This mechanism serves to minimize the number of words necessary for communicating all possible intended meanings. This is because it allows words to be used in many different ways, rather than requiring a different word for every possible meaning.

\section{The Semantic-Episodic Distinction}

Tulving (1972) proposed a distinction between episodic and semantic memories. Episodic memories represent autobiographical experiences, that is, events coded by space and time. In contrast, semantic memories represent our knowledge of the world and the meanings of words. ${ }^{4}$ Although most investigators have not argued for physically separate memories in the brain, many have agreed that there may be different representations and processes associated with each memory type. Recently, this view has come under attack. J. R. Anderson and Ross (1980) and McKoon and Ratcliff (1979) have argued that episodic and semantic memories may be similarly represented and subject to the same processes.
Barsalou and Bower (Note 1) further argue that the CI-CD distinction is problematic for the semanticepisodic distinction. If CI properties are automatized, and if practice results in automaticity, then particular processing episodes determine the accessibility of semantic memories. Similarly, $C D$ properties are $C D$ because there have not been many episodes in which the property and the respective word were simultaneously processed. Since the availability of semantic information depends directly on episodic information, it is not clear that two types of memories are needed when one would probably be sufficient.

Barsalou and Bower (Note 1) discuss specific ways in which particular processing episodes may affect the psychology of lexical semantics. To start with, a word can refer to different kinds of instances. "Car" can refer to cars with or without air conditioning. Consequently, the accessibility of "air conditioning" should depend on the type of car someone is used to. In general, properties of a concept not typically found for familiar referents may become $C D$ through disuse. In contrast, properties typically encountered are more likely to become CI. Analogously, particular uses of an object may vary in accessibility. Someone who has just been to a circus may be more likely to categorize "chair" as something to fight lions with. In general, encoding a particular episode in which an object is put to atypical use may make that use more accessible, at least temporarily.

It should be pointed out that CI properties are not necessarily more semantic than $\mathrm{CD}$ properties. Episodes can be CI (e.g., "doberman pincher" may always activate a particular, well rehearsed episode of being bitten by one of these dogs), and semantic properties can be CD (e.g., properties that are usually irrelevant for an object, such as "floats" for "basketball"). The primary difference between $\mathrm{CI}$ and $\mathrm{CD}$ information is simply the means by which they are activated: CI information is activated by the word for a concept, whereas CD information is activated by relevant contexts in which the word is encoded. As suggested by Barsalou and Bower (Note 1), this difference in accessibility is a function of the frequency and recency of processing episodes, regardless of whether the information is an episode, a semantic feature, an affect, or some other type of information.

Finally, E. V. Clark and H. H. Clark (1979) have shown that certain innovative uses of words can result in new meanings for those words. Computing these novel meanings often requires retrieving a particular episode. Consider their example of "teapotting." Suppose someone named Max has a strange habit of rubbing a teapot on the backs of people's legs. Imagine that two people had seen Max do this before, and one of them said, "Max is in trouble, he just teapotted a policeman." The listener would compute the meaning of "teapotted" by retrieving the relevant episodes, even though he or she has never heard the word used that way. Clearly, this example illustrates the necessity of using episodic information to arrive at the speaker's intended mean- 
ing. There is no linguistic rule that could generate the exact meaning intended by the speaker in this situation. Instead, the specifics of the meaning are derived from the structure of the relevant episodes. With recurrent uses of "teapot" in this manner, however, the new meaning could eventually be abstracted away from the particulars of episodes and become CI. Thus, particular processing episodes not only enable comprehension of certain linguistic innovations but are also responsible for the respective word senses' becoming well established in memory. In general, changes in word meanings over time may often be the result of changes in the accessibility of $\mathrm{CI}$ and $\mathrm{CD}$ properties.

\section{REFERENCE NOTES}

1. Barsalou, L. W., \& Bower, G. H. A priori determinants of a concept's highly accessible information. Paper presented at the annual meeting of the American Psychological Association, Montreal, September 1980.

2. Barsalou, L. W. Ad hoc categories and cross-classification. Unpublished manuscript, 1981.

3. Glucksberg, S., Dial, P. G., \& Bookin, H. B. On understanding nonliteral speech: Can people ignore metaphors? Manuscript in preparation, 1980.

\section{REFERENCES}

Anderson, J. R., \& Ross, B. H. Evidence against the semanticepisodic distinction. Journal of Experimental Psychology: Human Learning and Memory, 1980, 6, 441-466.

Anderson, R. C., \& Ortony, A. On putting apples into bottlesA problem of polysemy. Cognitive Psychology, 1975, 7, 167-180.

Andergon, R. C., Pichert, J. W., Goetz, E. T., Schallert, D. L., Stevens, K. V., \& Trollip, S. R. Instantiation of general terms. Journal of Verbal Learning and Verbal Behavior, 1976, 15, 667-679.

Barclay, J. R., Bransford, J. D., Franks, J. J., McCarrell, N. S., \& Nitscr, K. Comprehension and semantic flexibility. Journal of Verbal Learning and Verbal Behavior, 1974, 13, $471-481$.

Battig, W. F., \& Montague, W. E. Category norms for verbal items in 56 categories: A replication and extension of the Connecticut category norms. Journal of Experimental Psychology, 1969, 80(Whole No. 3, Pt. 2).

Bowen, G. H. Stimulus-sampling theory of encoding variability. In A. W. Melton \& E. Martin (Eds.), Coding processes in human memory. Washington, D.C: Winston, 1972.

Camp, C. J., Lachman, J. L., \& Lachman, R. Evidence for direct-access and inferential retrieval in question-answering. Journal of Verbal Learning and Verbal Behavior, 1980, 19, 583-596.

Clark, E. V., \& Clark, H. H. When nouns surface as verbs. Language, 1979, 55, 767-811.

Clark, H. H. The language-as-fixed-effect fallacy: A critique of language statistics in psychological research. Journal of Verbal Learning and Verbal Behavior, 1973, 12, 335-359.

Collins, A. M., \& Quillian, M. R. Retrieval time from semantic memory. Journal of Verbal Learning and Verbal Behavior, $1969,8,240-247$.

Conrad, C. Cognitive economy in semantic memory. Journal of Experimental Psychology, 1972, 92, 149-154.

Conrad, C. Some factors involved in the recognition of words. In J. W. Cotton \& R. L. Klatzky (Eds.), Semantic factors in cognition. Hillsdale, N.J: Erlbaum, 1978.

Duncke R, K. On problem solving. Psychological Monographs, 1945, 58(Whole No. 270).
Estes, W. K. Statistical theory of spontaneous recovery and regression. Psychological Review, 1955, 62, 145-154.

Estes, W. K. The statistical approach to learning theory. In $\mathbf{S}$. Koch (Ed.), Psychology: A study of a science (Vol. 2). New York: McGraw-Hill, 1959.

Foss, D. J., Cirilo, R. K., \& Blank, M. A. Semantic facilitation and lexical access during sentence processing: An investigation of individual differences. Memory \& Cognition, 1979, 7, 346-353.

Glass, A. L., \& Holyoak, K. J. Alternative conceptions of semantic memory. Cognition, 1975, 3, 313-339.

Glucksbera, S., \& Danks, J. Effects of discriminative labels and of nonsense labels upon availability of novel function. Journal of Verbal Learning and Verbal Behavior, 1968, 7, 72-76.

Glucksberg, S., \& Weisberg, R. W. Verbal behavior and problem solving: Some effects of labeling in a functional fixedness problem. Journal of Experimental Psychology, 1966, 71, 659-664.

GuILFORD, J. P., \& Fruchter, B. Fundamental statistics in psychology and education. New York: McGraw-Hill, 1973.

Jacoby, L. L., Craik, F. I. M., \& BEGG, I. Effects of decision difficulty on recognition and recall. Journal of Verbal Learning and Verbal Behavior, 1979, 18, 585-600.

Katz, J. J., \& Postal, P. An integrated theory of linguistic descriptions. Cambridge, Mass: M.I.T. Press, 1964.

McCloskey, M., \& Guucksbero, S. Decision processes in verifying category membership statements: Implications for models of semantic memory. Cognitive Psychology, 1979, 11, 1-37.

McKoon, G., \& RATcliff, R. Priming in episodic and semantic memory. Journal of Verbal Learning and Verbal Behavior, $1979,18,463-480$.

NEELY, J. H. Semantic priming and retrieval from lexical memory: Roles of inhibitionless spreading activation and limited-capacity attention. Journal of Experimental Psychology: General, 1977, 106, 226-254.

Olson, D. R. Language and thought: Aspects of a cognitive theory of semantics. Psychological Review, 1970, 77, 257-273.

Ortony, A. Beyond literal similarity. Psychological Review, 1979, 86, 161-180.

Posner, M. I., \& SNyder, C. R. R. Attention and cognitive control. In R. L. Solso (Ed.), Information processing and cognition: The Loyola symposium. Hillsdale, N.J: Erlbaum, 1975.

Rips, L. J., Shoben, E. J., \& Smith, E. E. Semantic distance and the verification of semantic relations. Journal of Verbal Learning and Verbal Behavior, 1973, 12, 1-20.

Rosch, E. H. Cognitive representations of semantic categories. Journal of Experimental Psychology: General, 1975, 104, 192-233.

Rosch, E. H., \& MErvis, C. B. Family resemblances: Studies in the internal structure of categories. Cognitive Psychology, 1975, 7, 573-605.

Rosch, E. H., Mervis, C. B., Gray, W. D., Johnson, D. M., \& Boyes-Braem, P. Basic objects in natural categories. Cognitive Psychology, 1976, 8, 382-439.

Shiffrin, R. M., \& Schneider, W. Controlled and automatic human information processing: II. Perceptual learning, automatic attending, and a general theory. Psychological Review, $1977,84,127-190$.

Smith, E. E., Shoben, E. J., \& RiPs, L. J. Structure and process in semantic memory: A featural model for semantic decisions. Psychological Review, 1974, 81, 214-241.

Swinney, D. A. Lexical access during sentence comprehension: (Re)Consideration of context effects. Journal of Verbal Learning and Verbal Behavior, 1979, 18, 645-659.

Tanenhaus, M. K., Leiman, J. M., \& Seidenberg, M. S. Evidence for multiple stages in the processing of ambiguous words in syntactic contexts. Journal of Verbal Learning and Verbal Behavior, 1979, 18, 427-440.

TABossi, P., \& Johnson-Laird, P. N. Linguistic context and the priming of semantic information. Quarterly Journal of Experimental Psychology, 1980, 32, 595-603. 
Tulving, E. Episodic and semantic memory. In E. Tulving \& W. Donaldson (Eds.), Organization and memory. New York: Academic Press, 1972.

Tulving, E., \& Thompson, D. M. Encoding specificity and retrieval processes in episodic memory. Psychological Review. 1973, 80, 352-373.

WINER, B. J. Statistical principles in experimental design. New York: McGraw-Hill, 1971.

\section{NOTES}

1. It should be pointed out that this proposal regarding the existence of $\mathrm{CI}$ and $\mathrm{CD}$ properties is not a theory of meaning. It simply addresses one aspect of concepts, namely, the accessibility of properties.

2. The CI control and CD unrelated-context items both have weakly related subject relations and unrelated predicate relations; analogously, the CD control and CI unrelated-context items both have highly related subject relations and unrelated predicate relations. It is not possible, however, to pool the laten- cies within these two sets of items, since this would make comparisons between unrelated-and related-context items impossible. The proper way to compare related and unrelated contexts is to observe latencies for the same properties under different context conditions. Pooling violates this design, since latencies for the control properties would be included in the unrelatedcontext conditions but not in the related-context conditions.

3. As discussed earlier, these senses may not be strictly CD. Even though context is required to converge on a particular sense, many may easily come to mind in no context (Swinney, 1979; Tanenhaus et al., 1979). Consequently, the primary senses of an ambiguous word may lie in the middle ground between CI and $C D$ properties. These senses are $C D$ in the weaker sense that they are attenuated or strengthened by context once their linguistic form has au tomatically activated them.

4. This use of "semantic" is nonstandard, since "semantics" is typically used to refer only to the meanings of words.

(Received for publication January 8, 1981; revision accepted August 13, 1981.) 